\title{
Artificial Pancreas as an Effective and Safe Alternative in Patients with Type 1 Diabetes Mellitus: A Systematic Review and Meta-Analysis
}

\author{
Xia Dai $\cdot$ Zu-chun Luo $\cdot$ Lu Zhai $\cdot$ Wen-piao Zhao $\cdot$ Feng Huang (iD
}

Received: April 13, 2018 / Published online: May 9, 2018

(c) The Author(s) 2018
Methods: Electronic databases were carefully searched for English publications comparing artificial pancreas with its control group. Overall daytime and nighttime glucose parameters were considered as the endpoints. Data were evaluated by means of weighted mean differences (WMDs) and 95\% confidence intervals (CIs) generated by RevMan 5.3 software.

Results: A total number of 354 patients were included. Artificial pancreas significantly maintained a better mean concentration of glucose (WMD $-1.03,95 \%$ CI -1.32 to $-0.75 ; P=$ $0.00001)$. Time spent in the hypoglycemic phase was also significantly lower (WMD $-1.23,95 \%$ CI -1.56 to $-0.91 ; P=0.00001)$. Daily insulin requirement also significantly favored artificial pancreas (WMD - 3.43, 95\% CI -4.27 to -2.59 ; $P=0.00001)$. Time spent outside the euglycemic phase and hyperglycemia phase (glucose $>10.0$ $\mathrm{mmol} / \mathrm{L}$ ) also significantly favored artificial pancreas. Also, the numbers of hypoglycemic events were not significantly different.

Conclusion: Artificial pancreas might be considered an effective and safe alternative to be used during a 24-h basis in patients with T1DM.

Keywords: Artificial pancreas; Glucose control; Type 1 diabetes mellitus
Abbreviations
T1DM Type 1 diabetes mellitus
WMD Weight mean difference
CIs Confidence intervals 


\section{INTRODUCTION}

Type 1 diabetes mellitus (T1DM) is still a major concern in this new era. This chronic disorder occurs when beta cells of the pancreas are destroyed by autoimmune antibodies at a younger age (childhood) or it can even develop in adults, during their late 30s or 40s [1]. Insulin injection has been the main treatment in these patients with T1DM [2]. However, despite concerted efforts of patients and physicians/caregivers, control of blood glucose has often been difficult to achieve [3].

With the development of new techniques and devices in clinical medicine, continuous glucose monitoring has significantly improved conditions of patients with T1DM [4]. To further enhance glucose control in these patients, an artificial pancreas has recently been developed [5].

Even though this artificial pancreas has already been approved for use by the US Food and Drug Administration (FDA) [5], the existence and benefits of this "expected to be" effective device are not well known to the general population.

In this analysis, we aimed to systematically compare artificial pancreas with its control group in terms of effectiveness and safety, during a 24-h treatment of patients with T1DM.

\section{METHODS}

\section{Searched Databases}

The Medline database of medical publications, the Cochrane library of randomized controlled trials, and EMBASE database were carefully searched by the five authors for English publications comparing artificial pancreas with its control group (any control group). Reference lists of selective publications (most relevant ones) were also carefully reviewed for appropriate articles. In addition, official websites of specific journals such as Lancet Diabetes and Endocrinology, Diabetes Care, and Cardiovascular Diabetology were also searched for any relevant publication.

\section{Search Strategies}

During this process, we searched for the terms "artificial pancreas", "bionic pancreas", "closed loop glucose control", "type 1 diabetes mellitus", "diabetes mellitus", "glucose control", and "glucose monitoring", one at a time and in combination. Publications comparing artificial pancreas with its control group were considered relevant to this analysis.

\section{Inclusion Criteria}

Studies were included if they compared artificial pancreas with its control group (any relevant control group); they reported glucose control parameters or other outcomes related to glucose monitoring (during daytime and overnight/24$\mathrm{h}$ basis); they involved patients with T1DM.

\section{Exclusion Criteria}

Studies were excluded if they did not compare artificial pancreas with its control group; they did not report glucose monitoring parameters as their endpoints or they reported only daytime or overnight measurement, but not both in combination; they involved patients with type 2 diabetes mellitus instead of patients with T1DM; they were duplicates.

\section{Type of Participants, Endpoints, and Follow-Up Periods}

This analysis included patients with T1DM.

The endpoints (during daytime and nighttime) which were analyzed included:

(a) Mean and median glucose concentration.

(b) Time spent outside the euglycemic phase (outside the glucose range 3.90 to $8.0 \mathrm{mmol} / \mathrm{L}$ ).

(c) Time spent in the hypoglycemia phase (blood glucose $<3.9 \mathrm{mmol} / \mathrm{L}$ ).

(d) Insulin required/delivered per day.

(e) Time spent in the hyperglycemia phase (blood glucose $>10.0 \mathrm{mmol} / \mathrm{L}$ ).

(f) Number of hypoglycemic events. 
The follow-up period ranged from less than 1 week to 2 months.

\section{Data Extraction, Review, and Statistical Analysis}

After the search process, which was conducted in accordance with the PRISMA guideline [6], the same reviewers assessed the titles and abstracts and independently selected the most suitable articles which satisfied the inclusion and exclusion criteria of this analysis and then data were extracted. The studies which were included in this analysis were judged as having low to moderate risk of bias [7].

This is a meta-analysis and therefore inconsistency across the studies was evident [8]. Hence, heterogeneity was assessed by two statistical methods:

(a) The $Q$ statistic test, whereby a $P$ value less or equal to 0.05 was considered statistically significant.

(b) The $I^{2}$ statistic test; a high percentage value indicated high heterogeneity (whereby a random effects model was used) and low percentage value denoted low heterogeneity (whereby a fixed effects model was used).

Since continuous data was used in this analysis, i.e., mean and standard deviation (SD), data were evaluated by means of weighted mean differences (WMDs) and 95\% confidence intervals (CIs). In case the $\mathrm{SD}$ value was not provided, but a $p$ value was given, $S D$ was calculated using the formula $\mathrm{SD}=\sqrt{ } n \times p \times(1-$ $p$ ). The analysis was carried out by RevMan 5.3 software.

Publication bias was assessed by visually observing funnel plots.

\section{Compliance with Ethics Guidelines}

This meta-analysis is based on previously conducted studies and does not contain any studies with human participants or animals performed by any of the authors.

\section{RESULTS}

\section{Search Outcomes, Main and Baseline Features of the Studies}

Eight studies [9-16] were selected to be used in this analysis as shown in Fig. 1.

Table 1 summarizes the main features of the studies included in this analysis. As per the criteria of this analysis which required an experimental and a control group, a total of 354 patients were included (177 patients in each group) as shown in Table 1.

The baseline features are reported in Table 2.

\section{Analysis Results}

Results of this current analysis showed artificial pancreas to significantly maintain a better mean concentration of glucose with WMD $-1.03,95 \% \mathrm{CI}-1.32$ to $-0.75 ; P=0.00001$, $I^{2}=46 \%$ compared to the control group during a 24-h basis. The median glucose concentration was similar in both groups with WMD -0.30 , $95 \%$ CI -1.03 to $0.44 ; P=0.43, I^{2}=0 \%$ as shown in Fig. 2.

In addition, the time spent in the hypoglycemic phase (glucose $<3.9 \mathrm{mmol} / \mathrm{L}$ ) also significantly favored artificial pancreas with

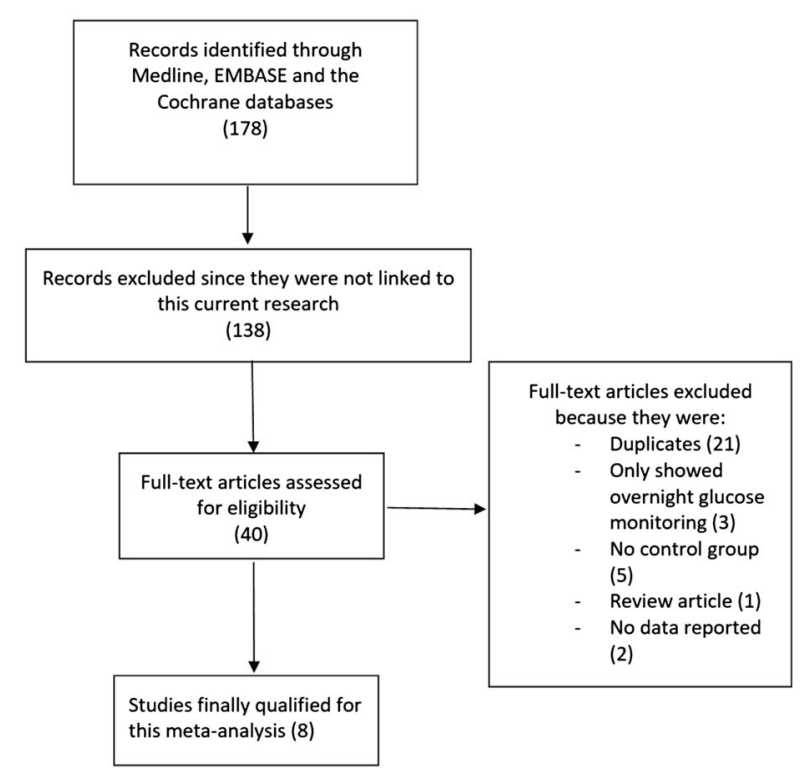

Fig. 1 Flow diagram representing the study selection 
Table 1 Main features of the studies included

\begin{tabular}{llccc}
\hline Studies & Type of study & $\begin{array}{l}\text { Patients with AP } \\
(\boldsymbol{n})\end{array}$ & $\begin{array}{l}\text { Patients in control group } \\
(\boldsymbol{n})\end{array}$ & $\begin{array}{l}\text { Total patients } \\
(\boldsymbol{n})\end{array}$ \\
\hline Blauw [9] & Randomized crossover & 5 & 5 & 10 \\
Kropff [10] & Randomized crossover trial & 32 & 32 & 64 \\
Renard [11] & $\begin{array}{l}\text { Single-arm non-randomized } \\
\text { extension }\end{array}$ & 20 & 20 & 40 \\
Thabit [12] & Randomized crossover & 24 & 24 & 48 \\
Kovatchev [13] & Randomized crossover & 18 & 18 & 36 \\
El-Khatib [14] & Randomized crossover trial & 39 & 39 & 78 \\
Russell [15] & Randomized crossover & 20 & 20 & 40 \\
Russell [16] & Randomized crossover trial & 19 & 19 & 38 \\
Total patients & & 177 & 177 & 354 \\
$(n)$ & & & \\
\hline
\end{tabular}

$A P$ artificial pancreas

Table 2 Baseline features of the patients in the studies included

\begin{tabular}{llllll}
\hline Studies & $\begin{array}{l}\text { Mean age (years) } \\
\text { AP/C }\end{array}$ & $\begin{array}{l}\text { Male (\%) } \\
\text { AP/C }\end{array}$ & $\begin{array}{l}\text { HBA1c } \\
\text { AP/C }\end{array}$ & $\begin{array}{l}\text { BMI }\left(\mathbf{k g} / \mathbf{m}^{2}\right) \\
\text { AP/C }\end{array}$ & $\begin{array}{l}\text { DM duration (years) } \\
\text { AP/C }\end{array}$ \\
\hline Blauw [9] & - & - & - & - & - \\
Kropff [10] & $47.0 / 47.0$ & $56.0 / 56.0$ & $8.2 / 8.2$ & $25.1 / 25.1$ & $28.6 / 28.6$ \\
Renard [11] & $46.3 / 46.3$ & $45.0 / 45.0$ & $8.2 / 8.2$ & $24.9 / 24.9$ & $28.9 / 28.9$ \\
Thabit [12] & $43.0 / 43.0$ & $54.2 / 54.2$ & $8.1 / 8.1$ & $26.0 / 26.0$ & $29.0 / 29.0$ \\
Kovatchev [13] & - & - & - & - & - \\
El-Khatib [14] & $33.3 / 33.3$ & $46.0 / 46.0$ & $7.7 / 7.7$ & $25.9 / 25.9$ & $16.9 / 16.9$ \\
Russell [15] & $40.0 / 40.0$ & $40.0 / 40.0$ & $7.1 / 7.1$ & $25.0 / 25.0$ & $24.0 / 24.0$ \\
Russell [16] & $9.80 / 9.80$ & $32.0 / 32.0$ & $7.8 / 7.8$ & $17.8 / 17.8$ & $5.00 / 5.00$ \\
\hline
\end{tabular}

$A P$ artificial pancreas, $C$ control group, $H B A 1 c$ glycosylated hemoglobin, $B M I$ body mass index, $D M$ diabetes mellitus

WMD $-1.23,95 \%$ CI -1.56 to -0.91 ; $P=0.00001, I^{2}=19 \%$ meaning the patients experienced less time in the hypoglycemia phase with this artificial pancreas as compared to the control group (Fig. 2).

Daily insulin required (24-h basis) also significantly favored artificial pancreas with WMD -3.43 , 95\% CI -4.27 to -2.59 ; $P=0.00001$,
$I^{2}=0 \%$ compared to the control group, indicating that good glucose control was continuously maintained without requiring an excess of insulin (Fig. 2).

Also, the numbers of hypoglycemic events were not significantly different with WMD $-0.83,95 \% \mathrm{CI}-1.76$ to $0.10 ; P=0.08, I^{2}=0 \%$ (Fig. 2). 


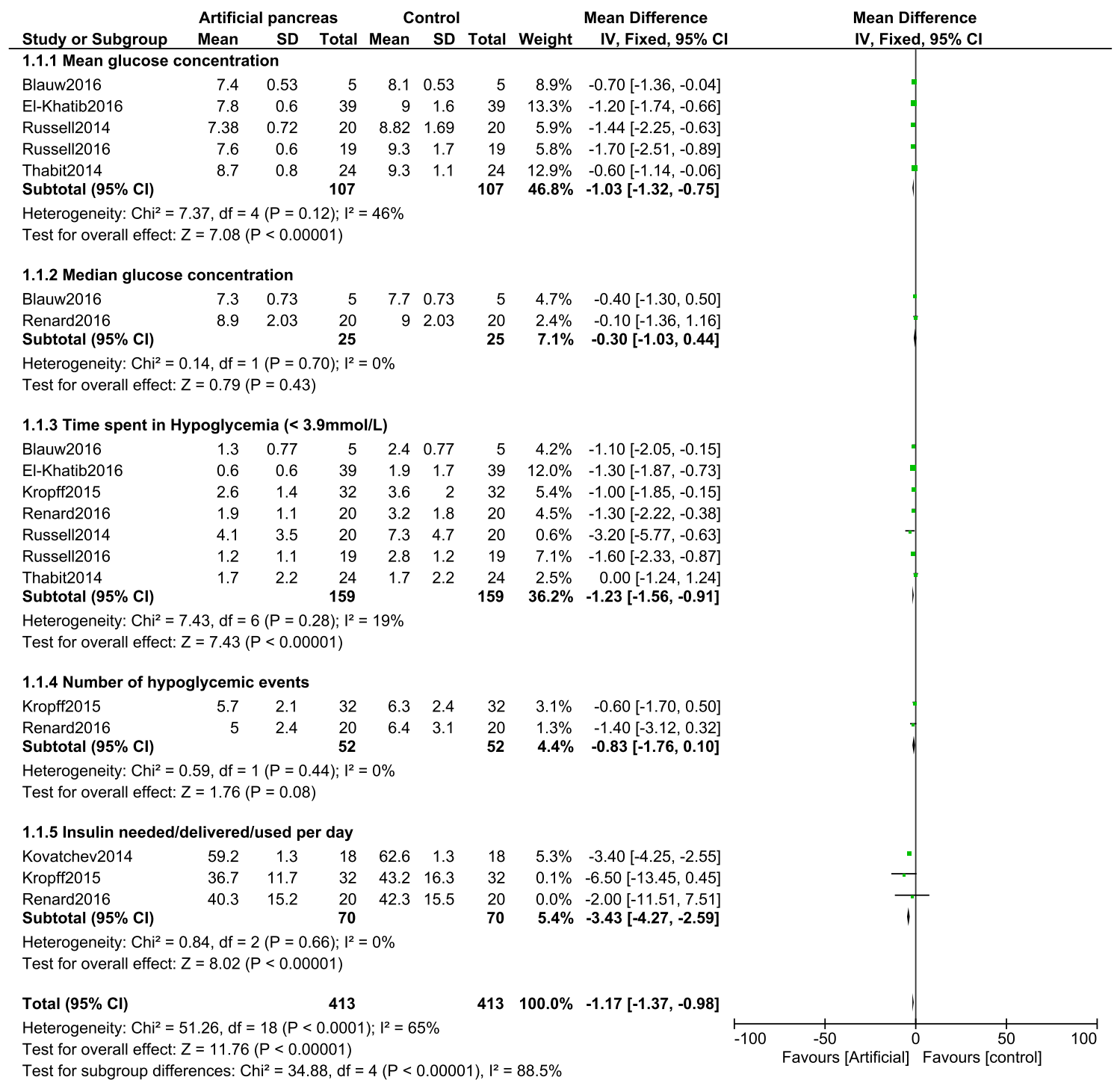

Fig. 2 Comparing artificial pancreas with the control group (part 1)

Time spent outside the euglycemic phase (24 h-basis) also significantly favored artificial pancreas with WMD $-6.28,95 \% \mathrm{CI}-10.67$ to $-1.88 ; P=0.005$. This meant that most of the time, patients using artificial pancreas were in the euglycemic phase (neither experiencing hypoglycemia nor hyperglycemia) as shown in Fig. 3. However, the results were moderately heterogeneous.

Moreover, the time spent in the hyperglycemia phase (glucose $>10.0 \mathrm{mmol} / \mathrm{L}$ ) also significantly favored artificial pancreas with WMD -13.20 , $95 \%$ CI -16.47 to -9.94 ;
$P=0.00001$ meaning that patients using artificial pancreas hardly suffered any hyperglycemic stage as shown in Fig. 3. However, this result was also moderately heterogeneous.

\section{DISCUSSION}

The use of a fully integrated artificial pancreas in patients with T1DM was previously demonstrated [17].

The current results showed artificial pancreas to be significantly more effective compared to its control group in terms of glucose 


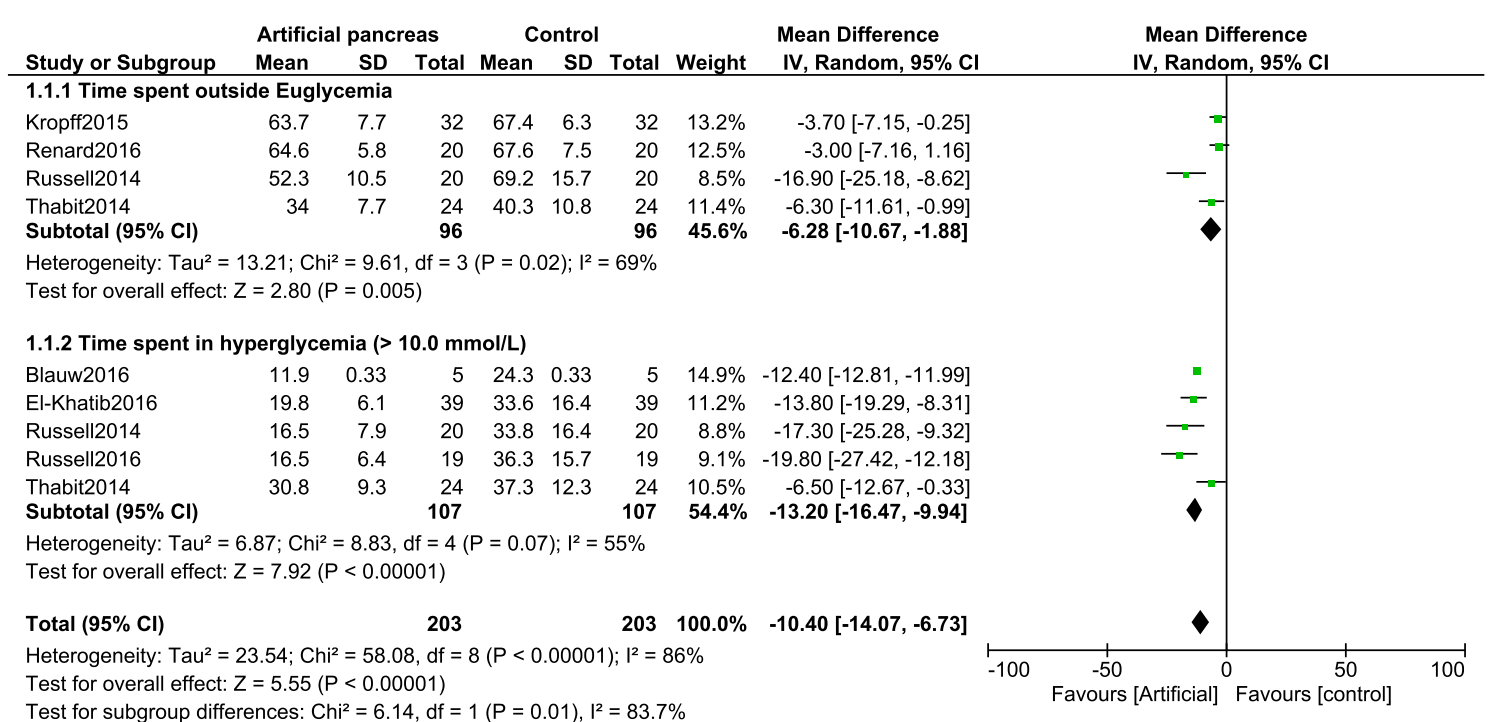

Fig. 3 Comparing artificial pancreas with the control group (part 2)

concentration, time spent in the hypoglycemic phase, and insulin delivery during a 24 -h period. Artificial pancreas was also safer to use owing to its association with a significantly lower time period in the hyperglycemia phase, its significant maintenance of a longer euglycemic period, and its lack of association with any significantly higher episode of hypoglycemic event compared to its control.

Similarly, Hovorka et al. showed that artificial pancreas improved overnight control of glucose level and decreased the rate of nocturnal hypoglycemia in patients with T1DM within a study time period of 3 months [18]. Another multicenter study showed this artificial pancreas to be very effective and safe to use in patients with T1DM [19]. Similarly, through a multicenter 6-month trial of 24/7 automated insulin delivery in 2014, Kovatchev et al. recently showed closed-loop control technology to have matured and to appear safe for longterm use in patients with T1DM [20].

This new device was even considered effective in pediatric participants. Weinzimer et al. recently demonstrated fully automated closedloop insulin delivery versus semi-automated hybrid control in pediatric candidates with T1DM [21]. Insulin delivery using artificial pancreas was further illustrated in the Virginia experience, wherein the participants were studied twice, once using their personal openloop technique, and then a second time using the closed-loop (artificial) system [22]. Other research further complemented the closed-loop insulin delivery technique $[23,24]$.

Further improvement is being considered in relation to this artificial pancreas [25]. Also, useful tools have already been devised to improve the assessment of glycemic variability in patients with artificial pancreas [26].

\section{LIMITATIONS}

This analysis also has limitations: (a) The number of participants was extremely limited; however, when compared to other previously published studies, this analysis included a large number of patients. (b) The different follow-up time periods could have had an impact on the results obtained. (c) The range of the euglycemic phase was supposed to be a glucose level ranging between 3.9 and $8.0 \mathrm{mmol} / \mathrm{L}$; however, a few studies recorded a glucose level varying between 4.4 and $8.0 \mathrm{mmol} / \mathrm{L}$ or 3.9 to $10.0 \mathrm{mmol} / \mathrm{L}$ which might have contributed to the moderate level of heterogeneity in this particular subgroup. (d) The inclusion of one non-randomized study might have introduced bias, contributing to the limitations in this analysis. (e) The control groups were not similar 
in all the studies, which might be another limitation of this analysis. (f) Utilizing sensor augmented pump as the control group is the current clinical golden standard which artificial pancreas needs to be able to outperform if clinically relevant. So, another limitation of this study might be the lack of an analysis strictly dealing with studies comparing artificial pancreas and sensor-augmented pump. However, the number of studies reporting this control was too small.

\section{CONCLUSION}

According to the results of this analysis, artificial pancreas might be considered an effective and safe alternative to be used during a 24-h basis in patients with T1DM. Several benefits of the artificial pancreas in maintaining and improving glucose levels were observed in comparison to its control. Nevertheless, a major shortcoming of this analysis is the extremely limited number of patients analyzed.

\section{ACKNOWLEDGEMENTS}

Funding. This research was supported by National Natural Science Foundation of China (No. 81560046), Guangxi Natural Science Foundation (No. 2016GXNSFAA380002), Scientific Project of Guangxi Higher Education (No. KY2015ZD028), Science Research and Technology Development Project of Qingxiu District of Nanning (No. 2016058), and Lisheng Health Foundation pilotage fund of Peking (No. LHJJ20158126). No funding or sponsorship was received for the publication of this article. The article processing charges were funded by the authors.

Authorship. All named authors meet the International Committee of Medical Journal Editors (ICMJE) criteria for authorship for this article, take responsibility for the integrity of the work as a whole, and have given their approval for this version to be published.
Authorship Contributions. Xia Dai, Zuchun Luo, Lu Zhai, Wen-piao Zhao, and Feng Huang were responsible for the conception and design, acquisition of data, analysis and interpretation of data, drafting the initial manuscript and revising it critically for important intellectual content. Xia Dai wrote the final manuscript. All the authors approved the manuscript as it is.

Disclosures. Xia Dai, Zu-chun Luo, Lu Zhai, Wen-piao Zhao, and Feng Huang declare that they have nothing to disclose. They do not have any personal, financial, commercial, or academic conflicts of interest.

Compliance with Ethics Guidelines. This meta-analysis is based on previously conducted studies and does not contain any studies with human participants or animals performed by any of the authors.

Data Availability. All data generated or analyzed during this study are included in this published article.

Open Access. This article is distributed under the terms of the Creative Commons Attribution-NonCommercial 4.0 International License (http://creativecommons.org/licenses/ by-nc/4.0/), which permits any noncommercial use, distribution, and reproduction in any medium, provided you give appropriate credit to the original author(s) and the source, provide a link to the Creative Commons license, and indicate if changes were made.

\section{REFERENCES}

1. Zayed H. Genetic epidemiology of type 1 diabetes in the 22 Arab countries. Curr Diab Rep. 2016;16(5):37.

2. Hurren KM, O'Neill JL. Pharmacodynamic and pharmacokinetic evaluation of insulin glargine U300 for the treatment of type 1 diabetes. Expert Opin Drug Metab Toxicol. 2016;12(12):1521-6.

3. McEwan P, Bennett H, Fellows J, Priaulx J, Bergenheim $\mathrm{K}$. The health economic value of changes in 
glycaemic control, weight and rates of hypoglycaemia in type 1 diabetes mellitus. PLoS One. 2016;11(9):e0162441.

4. Ramirez-Rincon A, Hincapie-García J, Arango CM, et al. Clinical outcomes after 1 year of augmented insulin pump therapy in patients with diabetes in a specialized diabetes center in Medellín, Colombia. Diabetes Technol Ther. 2016;18(11):713-8.

5. Voelker R. "Artificial pancreas" is approved. JAMA. 2016;316(19):1957.

6. Higgins JPT, Altman DG. Chapter 8: Assessing risk of bias in included studies. In: Higgins JPT, Green S, editors. Cochrane handbook for systematic reviews of interventions. Chichester: Wiley; 2008. p. 187-241.

7. Liberati A, Altman DG, Tetzlaff J, et al. The PRISMA statement for reporting systematic reviews and meta-analyses of studies that evaluate healthcare interventions: explanation and elaboration. BMJ. 2009;339:b2700.

8. Sterne JA, Egger M, Smith GD. Systematic reviews in health care: investigating and dealing with publication and other biases in meta-analysis. BMJ. 2001;323(7304):101-5.

9. Blauw H, van Bon AC, Koops R, DeVries JH. Performance and safety of an integrated bihormonal artificial pancreas for fully automated glucose control at home. Diabetes Obes Metab. 2016;18(7):671-7.

10. Kropff J, Del Favero S, Place J, et al. 2 month evening and night closed-loop glucose control in patients with type 1 diabetes under free-living conditions: a randomised crossover trial. Lancet Diabetes Endocrinol. 2015;3(12):939-47.

11. Renard E, Farret A, Kropff J, et al. Day-and-night closed-loop glucose control in patients with type 1 diabetes under free-living conditions: results of a single-arm 1-month experience compared with a previously reported feasibility study of evening and night at home. Diabetes Care. 2016;39(7):1151-60.

12. Thabit H, Lubina-Solomon A, Stadler M, et al. Home use of closed-loop insulin delivery for overnight glucose control in adults with type 1 diabetes: a 4-week, multicentre, randomised crossover study. Lancet Diabetes Endocrinol. 2014;2(9):701-9.

13. Kovatchev BP, Renard E, Cobelli C, et al. Safety of outpatient closed-loop control: first randomized crossover trials of a wearable artificial pancreas. Diabetes Care. 2014;37(7):1789-96.

14. El-Khatib FH, Balliro C, Hillard MA, et al. Home use of a bihormonal bionic pancreas versus insulin pump therapy in adults with type 1 diabetes: a multicentre randomised crossover trial. Lancet. 2016.

15. Russell SJ, El-Khatib FH, Sinha M, et al. Outpatient glycemic control with a bionic pancreas in type 1 diabetes. N Engl J Med. 2014;371(4):313-25.

16. Russell SJ, Hillard MA, Balliro C, Magyar KL, et al. Day and night glycaemic control with a bionic pancreas versus conventional insulin pump therapy in preadolescent children with type 1 diabetes: a randomised crossover trial. Lancet Diabetes Endocrinol. 2016;4(3):233-43.

17. Breton M, Farret A, Bruttomesso D, et al. Fully integrated artificial pancreas in type 1 diabetes: modular closed-loop glucose control maintains near normoglycemia. Diabetes. 2012;61(9):2230-7.

18. Hovorka R, Kumareswaran K, Harris J, et al. Overnight closed loop insulin delivery (artificial pancreas) in adults with type 1 diabetes: crossover randomised controlled studies. BMJ. 2011;13(342):d1855.

19. Del Favero S, Place J, Kropff J, et al. Multicenter outpatient dinner/overnight reduction of hypoglycemia and increased time of glucose in target with a wearable artificial pancreas using modular model predictive control in adults with type 1 diabetes. Diabetes Obes Metab. 2015;17(5):468-76.

20. Kovatchev B, Cheng P, Anderson SM, et al. Feasibility of long-term closed-loop control: a multicenter 6-month trial of $24 / 7$ automated insulin delivery. Diabetes Technol Ther. 2017;19(1):18-24.

21. Weinzimer SA, Steil GM, Swan KL, et al. Fully automated closed-loopinsulin delivery versus semiautomated hybrid control in pediatric patients with type1 diabetes using an artificial pancreas. Diabetes Care. 2008;31(5):934-9

22. Clarke WL, Anderson S, Breton M, et al. Closedloopartificial pancreas using subcutaneous glucose sensing and insulin delivery anda model predictive control algorithm: the Virginia experience. J Diabetes SciTechnol. 20091;3(5):1031-8.

23. Sharifi A, De Bock MI, Jayawardene D, et al.Glycemia, treatment satisfaction, cognition,and sleep quality in adults and adolescents with type 1 diabetes when using a closed-loop system overnight versus sensor-augmented pump with low-glucosesuspend function: a randomized crossover study. Diabetes Technol Ther. 2016;18(12):772-83.

24. Weisman A, Bai JW, Cardinez M, Kramer CK, Perkins BA. Effect of artificialpancreas systems on glycaemic control in patients with type 1 diabetes: a 
systematicreview and meta-analysis of outpatient randomised controlled trials. Lancet DiabetesEndocrinol. 2017;5(7):501-12

25. Gildersleeve R, Riggs SL, Cherñavvsky DR, Breton $\mathrm{MD}$, DeBoer MD. Improvingthe safety and functionality of an artificial pancreas system for use in youngerchildren: input from parents and physicians. Diabetes Technol Ther. 2017;19(11):660-74
26. Garcia A, Balo AK, Buckingham BA, Hirsch IB, Peyser TA. Application ofglycemic variability percentage: implications for continuous glucose monitorutilization and analysis of artificial pancreas data. Diabetes Technol Ther. 2017;19(12):699-706. 